\title{
Consumption trend and prescription pattern of opioid analgesics in China from 2006 to 2015
}

\author{
Wentong Fang, ${ }^{01}$ Tingting Liu, ${ }^{2}$ Zhongsheng Gu, ${ }^{1}$ Qian Li, ${ }^{1}$ Can Luo ${ }^{1}$
}

\begin{abstract}
- Additional material is published online only. To view please visit the journal online (http://dx.doi.org/ 10.1136/ejhpharm-2017001460).
\end{abstract}

${ }^{1}$ Department of Pharmacy, First Affiliated Hospital of Nanjing Medical University, Nanjing, China

${ }^{2}$ Department of Pharmacy, Nanjing General Hospital, Nanjing, China

\section{Correspondence to}

Wentong Fang, Department of Pharmacy, First Affiliated Hospital of Nanjing Medical University, Nanjing, Jiangsu 210029, China; fangwentong@jsph.org.cn

Received 21 November 2017 Revised 10 January 2018 Accepted 16 January 2018 Published Online First 27 January 2018

EAHP Statement 1: Introductory Statements and Governance.

\begin{abstract}
Objectives To investigate the consumption trend and prescription pattern of opioids in China from 2006 to 2015, and compare the results with those of selected countries and regions, and identify the pain relief level of China.
\end{abstract}

Methods Nine different opioid drugs were included in our survey, based on the whole population of China. Consumption of each type of opioid was calculated in grams in reports of the Chinese Food and Drug Administration and the International Narcotics Control Board. Then it was converted to the terms of defined daily doses and morphine equivalents. The correlation between consumption of opioids and gross domestic product, human development index and cancer incidence was analysed by Pearson's correlation test.

Results Defined daily doses increased by $7.89 \%$ per year since 2006, and reached 91.05 in 2015. Morphine equivalents increased by $10.57 \%$ per year since 2006 , and reached $7.24 \mathrm{~g}$ in 2015 . The average defined daily doses and morphine equivalents were 85.44 and $5.70 \mathrm{~g}$ respectively in 2006-2015. Annual defined daily doses of China were lower than the global average. Consumption was related to gross domestic product, but not human development index and cancer incidence. Furthermore, defined daily doses varied greatly in countries with similar gross domestic product or the human development index. Strong opioids or non-intravenous opioids always constituted the majority of opioids, and sustained an increasing tendency in consumption. The predominantly prescribed opioids were fentanyl and morphine. Consumption of oxycodone had a tendency to increase, whereas pethidine tended to decrease.

Conclusion Consumption of opioids has shown an increasing tendency and in the meantime the prescription pattern has changed, indicating the progress of pain relief in China, though it remained far lower than the average global level.

\section{INTRODUCTION}

Opioids are substances that act on opioid receptors to produce morphine-like effects. Opioids are commonly used for the control of clinical pain ${ }^{12}$ and have been identified by WHO as 'indispensable for the relief of pain and suffering', so that their availability 'must be' ensured. ${ }^{3}{ }^{4}$ However, consumption of opioids remains inadequate in some regions of the world, and many patients with moderate and severe pain have no access to the treatment needed. ${ }^{5}$ It was reported that in $2010,66 \%$ of the world population had virtually no opioid consumption, $10 \%$ was very low, 3\% low, $4 \%$ moderate and only $7.5 \%$ adequate. ${ }^{6}$ This discrepancy differs considerably among different countries and regions. The consumption was high in some European countries such as the UK, and in the USA and Canada. ${ }^{7}$ However, it was very low in some Asian countries such as India, Thailand and China. ${ }^{7}$ It has been reported that the average morphine equivalent (MEs) of China was $2.86 \mathrm{mg}$ per capita, while the average level of the top 20 countries in the human development index (HDI) was $216.7 \mathrm{mg}$ per capita in $2010 .^{6}$

The Chinese government and health organisations have made great efforts to address this situation. The Chinese government has issued 'the regulations on the administration of anaesthetic drugs and psychotropic drugs' in $2005,{ }^{8}$ which regulates the administration of opioids, ensures their legitimate medical utilisation, and prevents illegal abuse. The National Health and Family Planning Commission (NHFPC) of the People's Republic of China has launched the 'Good Pain Management Programme (GPM programme)' across the country since 2011..$^{9} 10$ The GPM programme emphasised the control of cancer pain and reasonable application of opioids. The NHFPC has also sponsored the training of professionals, publishing guidelines for pain management, distributing patient educational materials, and organising seminars or workshops throughout the country. ${ }^{11}$ As a result, some changes have recently been made in the consumption and prescription pattern of opioids. Hence the aim of this study is to investigate the consumption trend and prescription pattern of opioids in China from 2006 to 2015 , compare the results with those of selected regions and countries, and identify the pain relief level of China.

\section{METHODS}

\section{Data sources}

Eleven kinds of opioids are used on the Chinese mainland: morphine, fentanyl, oxycodone, codeine, opium, hydromorphone, hydrocodone, pethidine, dihydrocodeine, methadone and buprenorphine. Dextropropoxyphene, ketobemidone, tilidine and trimeperidine were not accessible in China. In our study, methadone and buprenorphine were excluded because their use for pain relief cannot be distinguished from their predominant treatment for dependence on illicit opioids. Therefore, consumption of morphine, fentanyl, oxycodone, codeine, opium, hydromorphone, hydrocodone, pethidine and dihydrocodeine was included and analysed in our study.

This study was based on the whole population of China. The Chinese Food and Drug Administration (FDA) and the International Narcotics Control Board (INCB) ${ }^{12}$ annually report the consumption of each type of opioid. They reported the 
consumption of opioids in grams. We estimate consumption in terms of defined daily doses (DDDs) and MEs.

The data for cancer incidence were reported by Chen et al. ${ }^{13} 14$ Population, gross domestic product (GDP) and HDI data were retrieved from the World Bank ${ }^{15}$ and the Human Development Report. ${ }^{16}$

\section{STATISTICAL ANALYSIS}

The DDD is a statistical unit defined by the WHO Collaborating Centre (WHOCC) for Drug Statistics Methodology. ${ }^{17}$ In our study, DDDs were shown as the values per million people per day and calculated with the following formula:

DDDs $=\left(\sum(\right.$ Total dose used $\left.) / \mathrm{DDD}\right) / 365 /$ population

$\mathrm{ME}$ is another unit to measure the consumption recommended by IAHPC. ${ }^{18} 19$ ME ratios are recommended by WHOCC for Drug Statistics Methodology. ${ }^{20}$ In our study, MEs are shown as the values per million people per day and calculated with the following formula:

MEs $=\left(\sum(\right.$ Total dose used $) *($ ME ratio $\left.)\right) / / 365 /$ population

GDP was shown as GDP per million people per day and calculated with the following formula:

GDP $=($ total GDP $) / 365 /$ population

Correlation of consumption of opioids and GDP, HDI and cancer incidence was analysed by applying Pearson's correlation test in SPSS version 21 (IBM Corporation, Armonk, New York, USA). $\mathrm{P}<0.05$ was considered a linear correlation.

\section{RESULTS}

\section{Opioid consumption had an increasing tendency}

DDDs have increased by $7.89 \%$ per year since 2006 and reached 91.05 in 2015. The DDD in 2015 was 1.98 times that in 2006 (figure 1A). MEs increased by $10.57 \%$ per year since 2006 and reached $7.24 \mathrm{~g}$ in 2015. The ME for 2015 was 2.47 times that in 2006 (figure 1B). The average DDD and ME were respectively 85.44 and $5.70 \mathrm{~g}$ in 2006-2015.

\section{Consumption of China is relatively low in the world}

We further compared opioid consumption in China with the average value for some other main regions of the world. In 2011-2013, the annual average DDD for China was 96, which was much lower than the global average value. Around the world, the DDD for China was much lower than the average level of North America, West and Central Europe, Oceania, Southeast Europe and South America, similar to the average level of East Europe and Central America, but higher than the average level for Africa and South Asia (table 1).

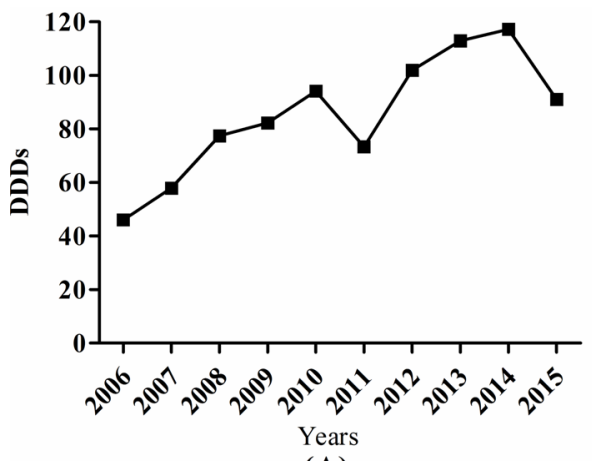

(A)
Table 1 The average defined daily doses (DDDs) of opioids in each main region in 2011-2013

\begin{tabular}{lc}
\hline Region & Average DDD $^{7}$ \\
\hline North America & 31453 \\
West and Central Europe & 9320 \\
Oceania & 9136 \\
\hline Southeast Europe & 1100 \\
South America & 347 \\
West Asia & 202 \\
\hline East and Southeast Asia & 189 \\
East Europe & 106 \\
Central America & 94 \\
Africa & 41 \\
South Asia & 11 \\
Global average level & 3027 \\
\hline
\end{tabular}

DDD is shown as the value per million people per day.

As an important country in East and Southeast Asia, the DDD for China was lower than the mean value of this region. It ranked sixth place among the 16 countries in this region. It was much lower than that of South Korea, Japan, Singapore, Brunei and Malaysia, but higher than other countries (table 2).

\section{Influence factors in the consumption of opioids}

GDP had a positive linear correlation with DDDs $\left(\mathrm{R}^{2}=0.84\right.$, $\mathrm{P}<0.0025)$ (figure $2 \mathrm{~A})$ and MEs $\left(\mathrm{R}^{2}=0.92, \quad \mathrm{P}<0.0002\right)$ (figure 2B) in 2006-2015. However, the DDDs of opioids varied greatly in countries with similar GDP (13-21 million dollars). ${ }^{15}$ The DDD for China was much lower than that of the Arab World, St Vincent and the Grenadines, Belarus, Serbia and Montenegro, almost the same level as Thailand, Macedonia, Cuba, Peru and Botswana, but higher than that of St Lucia, Iraq, Angola, Azerbaijan, Turkmenistan, the Maldives and Dominican Republic (online supplementary table S1).

The calculation formula for HDI has changed since $2009,{ }^{16}$ and the correlation between HDI and opioid consumption could not be measured (figure 2C,D). In countries of similar HDI $(0.68-0.75),{ }^{16}$ the consumption of opioids varied greatly. The consumption in China was much lower than that of Jordan, Colombia, Brazil, Bosnia and Herzegovina, almost the same level as Ecuador, Thailand, the former Yugoslav Republic of Macedonia and Peru, but higher than that of Suriname, Saint Lucia, Azerbaijan and Dominican Republic (online supplementary table S2).

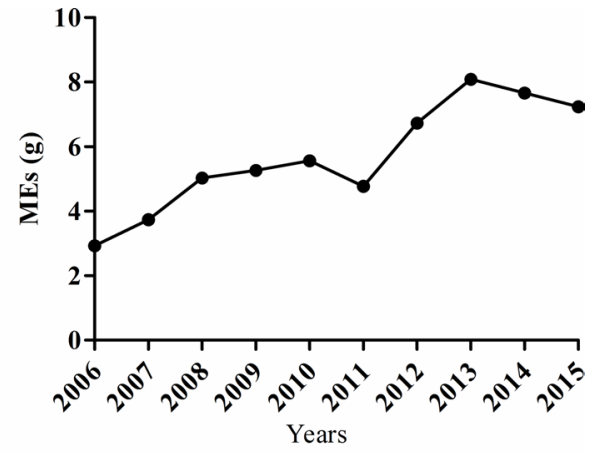

(B)

Figure 1 The consumption of opioid analgesics in China from 2006 to 2015. (A) The defined daily doses (DDDs) of opioid analgesics. (B) The morphine equivalents (MEs) of opioid analgesics. 


\begin{tabular}{|c|c|}
\hline Country & Average DDD $^{7}$ \\
\hline South Korea & 2244 \\
\hline Japan & 1192 \\
\hline Singapore & 435 \\
\hline Brunei & 219 \\
\hline Malaysia & 212 \\
\hline China & 96 \\
\hline Thailand & 85 \\
\hline Vietnam & 34 \\
\hline Mongolia & 29 \\
\hline North Korea & 26 \\
\hline Philippines & 21 \\
\hline Indonesia & 16 \\
\hline Laos & 7 \\
\hline Cambodia & 6 \\
\hline Myanmar & 0 \\
\hline Timor-Leste & 0 \\
\hline
\end{tabular}

DDD is shown as the value per million people per day.

Opioids are extensively used for the control of cancer pain. Age-standardised cancer incidence (ASR) changed little, ${ }^{13} 14$ while the consumption of opioids showed upward trends during the study period. Cancer ASR had no correlation with DDDs $\left(\mathrm{R}^{2}=0.03, \mathrm{P}=0.9464\right)$ (figure $\left.2 \mathrm{E}\right)$ and $\mathrm{MEs}\left(\mathrm{R}^{2}=0.24, \mathrm{P}=0.53\right.$ ) (figure 2F).

\section{Prescription pattern of opioids}

Strong opioids accounted for more than 90\% of the total consumption and had a tendency to increase. The DDDs of strong opioids in 2015 was twofold that in 2006. The DDDs of weak opioids remained low and changed little in 2006-2015 (figure 3A).

Consumption of non-intravenous opioids always constituted the majority of total consumption from 2006 to 2015. The DDDs of non-intravenous opioids had a tendency to increase. The DDDs of non-intravenous opioids in 2015 was 2.54 times that in 2006. The DDDs of intravenous opioids changed little in 2006-2015 (figure 3B).

Some changes have happened in the prescription pattern of opioids from 2006 to 2015. Consumption of fentanyl, which was the highest in 2006-2014, decreased to be lower than morphine and oxycodone in 2015. The DDD of morphine, which accounted for more than $20 \%$ of the total consumption, had a general tendency to rise in 2006-2015. Consumption of oxycodone was low in 2003-2011, but increased markedly in 2012-2015. The proportion of DDD of oxycodone constituted up to $27.70 \%$ of the total DDD in 2015 . Consumption of pethidine reduced since 2010 and was used by a minority in 2015. Consumption of other opioids such as codeine, opium, hydromorphone and hydrocodone remained low in 2006-2015 (figure 4).

\section{DISCUSSION}

For decades, morphine consumption has been proposed by $\mathrm{WHO}$ as an indicator of cancer pain management. ${ }^{21}$ The production and consumption of morphine have significantly increased in China from 1992 to $2011{ }^{8}$ However, over the past 20 years, additional opioids, such as fentanyl, hydromorphone and oxycodone, have been introduced in global and national markets. These types should be considered when studying the consumption of opioids. ${ }^{20}$ In our study, we included morphine, fentanyl, oxycodone, pethidine and hydromorphone as in previously reported studies, but we also included codeine, opium, hydrocodone and dihydrocodeine, which are still used in China. As shown in our study, the consumption of opioids had a general tendency to increase from 2006 to 2015 (figure 1), which reflected the progress in pain relief in China.

However, the opioid consumption of China was relatively low compared with the rest of the world. The DDDs of China was much lower than the average level of the whole world and most other regions (table 1), indicating that the pain relief level of China was relatively low compared with the rest of the world. Within East and Southeast Asia, the consumption of China was lower than the mean value of this region, and ranked sixth place of the 16 countries in this region (table 2), which indicates a medium level of pain relief in China. The study of Berterame et al also demonstrated the relatively low level of pain management in China.?

Many impediments could influence the consumption of opioids. The study by Berterame et al showed that GDP and HDI are important determinants of the consumption of opioids. ${ }^{7}$ In our study, consumption was correlated with GDP (figure 2A and B), but not with HDI. Furthermore, DDDs varied significantly in the countries with similar GDP (online supplementary table S1) or HDI (online supplementary table S2), meaning that these were not decisive measures of consumption.

The prevalence of health disorders warrants the use of certain drugs. ${ }^{7}$ Opioids are extensively used for the control of cancer pain. ${ }^{21}$ However, no correlation has been found (figure $2 \mathrm{E}$ and F). Berterame et al examined the relationship between cancer incidence and opioid consumption in countries worldwide and regionally, and found the relation shows a marked variation, as would be expected in view of the disparities in use between countries. ${ }^{7}$ So we considered that the increasing consumption of opioids in China might be caused by other factors.

Other impediments identified include reimbursement policies, prescription regulations and availability of pain services. ${ }^{1121} 22$ Reimbursement policies of opioids had improved in 2009. Sustained-release formulations of opioids, such as controlled-release morphine, controlled-release oxycodone and transdermal fentanyl, have been involved in healthcare since $2009 .^{23}$ This change in reimbursement policies might help in the availability of opioids.

The Chinese government has issued 'the regulation on the administration of anaesthetic drugs and psychotropic drugs' since $2005 .^{8}$ The regulation has been used in the administration of opioids to ensure their legitimate medical utilisation and to prevent illegal abuse. According to this regulation, the prescription dose for injection, other types of formulations and sustained-release formulations has been extended to a 3-day dose, a 7-day dose and a 15-day dose for cancer-related pain. ${ }^{8}$ This might have contributed to the increasing consumption of opioids.

Expansion of pain services may be a potential contributor to this increase. The NHFPC of the People's Republic of China has launched the 'GPM programme' across the country since $2011 .{ }^{9}$ A total of 87 hospitals have enrolled in this programme since $2012 .{ }^{10}$ The GPM programme emphasised the control of cancer pain and reasonable application of opioids. Yang $e \mathrm{al}^{24}$ revealed that the GPM programme could improve the pain management of patients with cancer and provide experience for improving cancer pain management in the future. 


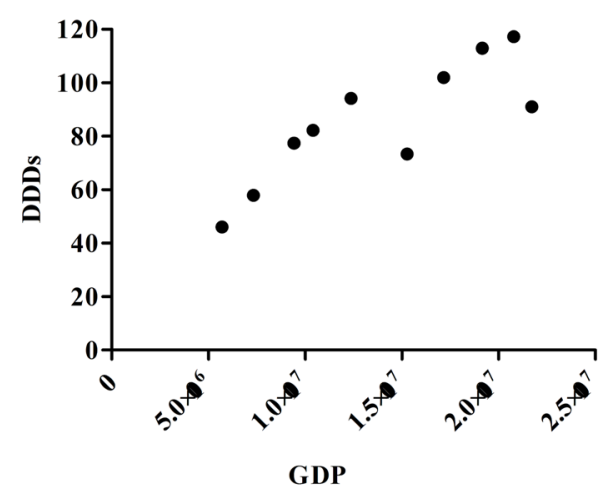

(A)

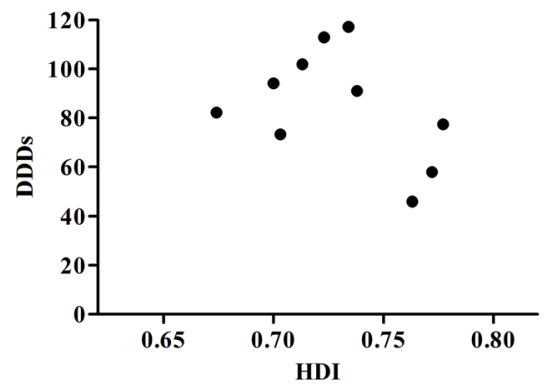

(C)

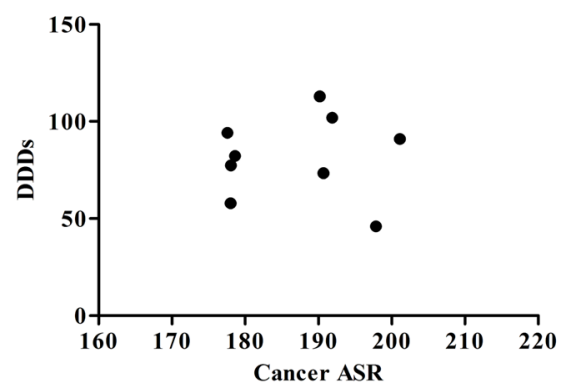

(E)

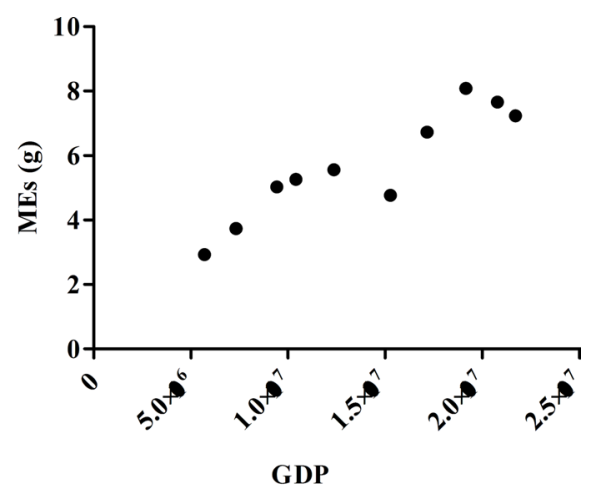

(B)

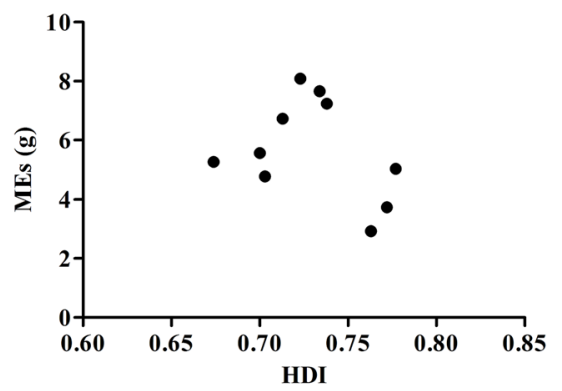

(D)

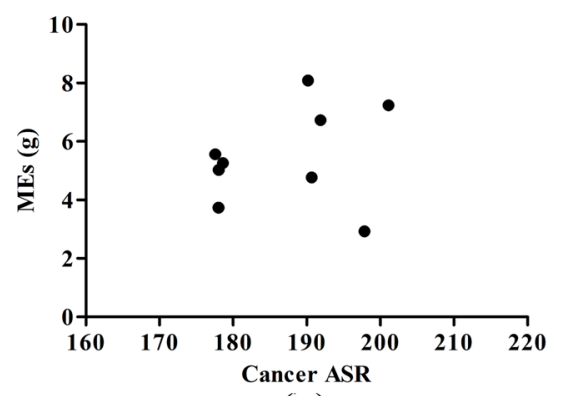

(F)

Figure 2 Analysis of influence factors of opioid consumption in China from 2006 to 2015. (A) Correlation analysis of defined daily doses (DDDs) and gross domestic product (GDP); (B) correlation analysis of morphine equivalents (MEs) and GDP; (C) correlation analysis of DDDs and human development index (HDI); (D) correlation analysis of MEs and HDI; (E) correlation analysis of DDDs and cancer age standardised rate (ASR); (F) correlation analysis of MEs and cancer ASR.

The GPM programme highlights the non-invasive administration of opioids, which was also emphasised by WHO's cancer pain ladder for adults. ${ }^{25}$ Therefore, consumption of non-iv opioids always took majority of the total consumption, and had an ascending tendency in 2006-2015 (figure 3B).

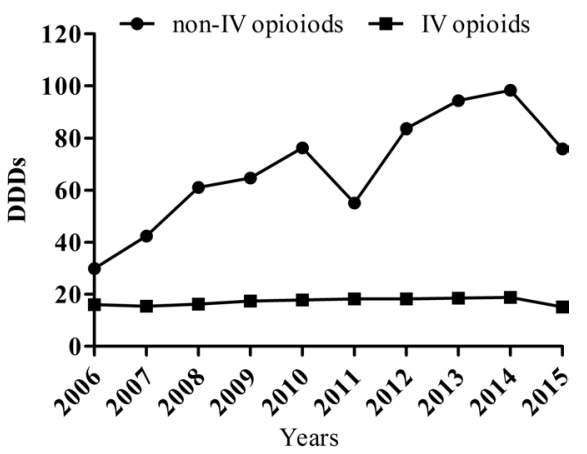

(B)

Figure 3 Consumption of opioid subtypes in China from 2006 to 2015. (A) Consumption of strong and weak opioids; (B)consumption of intravenous (IV) and non-IV opioid analgesics. DDD, defined daily dose. 


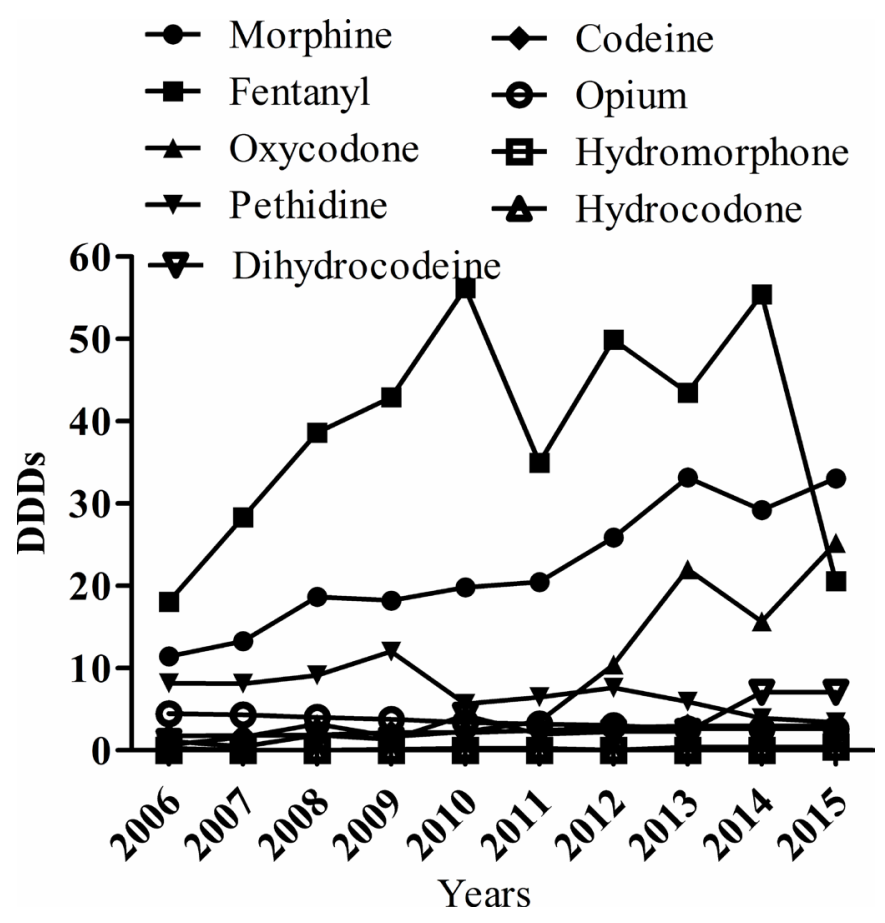

Figure 4 Consumption of each type of opioid in China from 2006 to 2015. DDD, defined daily dose.

Since 1986, the WHO three-step 'ladder' has recommended weak opioids for patients with moderate pain. ${ }^{26}$ Recent researches have revealed that passing directly from step I to step III of the WHO analgesic ladder is more efficient than the traditional three-step strategy for mild-moderate chronic cancer pain. ${ }^{25} 26$ Nowadays, the European Association for Palliative Care (EAPC) guidelines recommend small dose of strong opioids instead of weak opioids for patients with refractory moderate pain. ${ }^{27}$ The NCCN adult cancer pain guideline recommends opioids for all levels of pain. ${ }^{28}$ This could explain the overwhelming usage of strong opioids in 2006-2015 (figure 3A).

The prescription pattern of opioids for pain management has changed. For decades, morphine has made a large contribution to total opioid consumption in most countries. ${ }^{21}$ In our study, the DDD of morphine was lower than that of fentanyl in 2006-2014. Fentanyl was the most widely applied type in 2006-2014 (figure 4). Increased use of fentanyl has been reported in many countries such as the USA, Japan, Austrilia,

\section{What this paper adds}

What is already known on this subject

- The consumption of opioids was very low in China in 2010.

- The Chinese government and health organisations have made great efforts to address this situation.

\section{What this study adds}

- The consumption of opioids had an increasing tendency in China from 2006 to 2015, though it remained far lower than the average global level.

- The prescription pattern of opioids has changed in China from 2006 to 2015.

- The Chinese government has taken steps to emphasise the control of pain and expansion of pain services, but more needs to be done in the future.
Norway, Spain and Sweden. ${ }^{29}$ Transdermal fentanyl enables a slow increase of drug plasma level with a very long apparent half-life, and has fewer opioid-related gastrointestinal side effects, such as nausea, vomiting and constipation. ${ }^{30}$ In addition, the DDD for fentanyl used in this study was $0.6 \mathrm{mg}$, which was recommended by WHO and INCB, but the daily dose is usually $1.2-2.4 \mathrm{mg} /$ day in our practice. This might explain the large consumption of fentanyl in China.

Increased use of oxycodone has also been reported in many countries. $^{29}$ The rescue analgesic dose of controlled-release oxycodone is smaller than that of controlled-release morphine, while the occurrence rate of constipation, nausea and vomiting is lower than that of controlled-release morphine. ${ }^{31}$ In our study, the consumption of oxycodone increased significantly in 2012-2015, and constituted $27.70 \%$ of the total consumption in 2015 (Fig 7). Pethidine has a short duration of action and is limited to patients allergic to or unable to tolerate all other opioids in many countries including China. Routine and longterm use of pethidine is not recommended by health experts, who are concerned about its efficacy, toxicity and potential for abuse. ${ }^{19}$ A decrease in consumption of pethidine has been observed in many other countries such as Australia, Denmark and the USA. In our study, pethidine was one of the main types in 2006-2009, had a general tendency to decrease since 2010, and became a minority type in 2015. Consumption of other opioids such as codeine, opium, hydromorphone dihydrocodeine and hydrocodone were always low from 2006 to 2015 (figure 4). Briefly, the prescription pattern of opioids was generally reasonable.

\section{CONCLUSION}

The consumption of opioids had an increasing tendency and the prescription pattern has changed, indicating the progress in pain relief in China from 2006 to 2015, though it remained far lower than the average global level. The Chinese government has taken steps to emphasise the control of pain and expansion of pain services, but more needs to be done in the future.

Acknowledgements The work was supported by the Shire Biological Medicine Fund of Jiangsu Pharmaceutical Society (S201602)

Contributors WF: data calculation; data analysis; article design and writing. TL: manuscript revising. ZG: data check; statistical analysis. QL: data check; statistical analysis. CL: statistical analysis check.

Funding This study was funded by Shire Biological Medicine Fund of of Jiangsu Pharmaceutical Society.

Competing interests None declared.

Patient consent Obtained.

Provenance and peer review Not commissioned; externally peer reviewed.

(C) European Association of Hospital Pharmacists (unless otherwise stated in the text of the article) 2019. All rights reserved. No commercial use is permitted unless otherwise expressly granted.

\section{REFERENCES}

1 Raffa RB. On subclasses of opioid analgesics. Curr Med Res Opin 2014;30:2579-84.

2 Ghelardini C, Di Cesare Mannelli L, Bianchi E. The pharmacological basis of opioids. Clin Cases Miner Bone Metab 2015;12:219-21.

3 University of Wisconsin Pain \& Policy Studies Group. Do international model drug control laws provide for drug availability? I Pain Palliat Care Pharmacother 2009;23:145-52.

4 Lohman D, Schleifer R, Amon JJ. Access to pain treatment as a human right. BMC Med 2010;8:8.

5 Seya MJ, Gelders SF, Achara OU, et al. A first comparison between the consumption of and the need for opioid analgesics at country, regional, and global levels. J Pain Palliat Care Pharmacother 2011;25:6-18. 
6 Duthey B, Scholten W. Adequacy of opioid analgesic consumption at country, global, and regional levels in 2010, its relationship with development level, and changes compared with 2006. J Pain Symptom Manage 2014;47:283-97.

7 Berterame S, Erthal J, Thomas J, et al. Use of and barriers to access to opioid analgesics: a worldwide, regional, and national study. Lancet 2016;387:1644-56.

8 Gu W. Update on administration of anesthetics and psychoactive drugs for pain management in China. Acta Anaesthesiol Taiwan 2015;53:55-7.

9 National Health and Family Planning Commission of the People's Republic of China. 'Establishing good cancer pain management program'. http://www.nhfpc.gov.cn/ mohyzs/s3586/201104/51234.shtml (accessed 8 Jul 2016).

10 National Health and Family Planning Commission of the People's Republic of China. Hospitals enrolled in 'Good cancer pain management program'. http://www.nhfpc. gov.cn/mohyzs/s3586/201211/56217.shtml (accessed 8 Jul 2016).

11 Huang Y. Current status of pain management in China: an overview. Eur J Pain 2001;5 (Suppl A):67-71.

12 INCB. INCB Technical publications. International narcotics control board, 1995-2015. http://www.incb.org/incb/en/publications/technical-reports.html (accessed 8 Jul 2016).

13 Chen W, Zheng R, Baade PD, et al. Cancer statistics in China, 2015. CA Cancer J Clin 2016;66:115-32.

14 Chen W, Zheng R, Zhang S, et al. Report of cancer incidence and mortality in China, 2012J. China Cancer 2016;25:1-8.

15 The World Bank. GDP per capita (current US\$). http://data.worldbank.org/indicator/ NY.GDP.PCAP.CD (accessed 8 Jul 2016).

16 UNDP. Human Development Index and its components. http://hdr.undp.org/en/ composite/HDI (accessed 8 Jul 2016).

17 WHO Collaborating Centre for Drug Statistics Methodology. ATC/DDD Index. 2017. https://www.whocc.no/atc_ddd_index/ (accessed 8 Jul 2016).

18 Svendsen K, Mellbye A, Fredheim OMS, et al. 433 Monitoring the opioid consumption: Morphine-equivalent defined daily dose as a supplement to ddd. European Journal of Pain 2009;13:S130-1.

19 Svendsen K, Borchgrevink P, Fredheim O, et al. Choosing the unit of measurement counts: the use of oral morphine equivalents in studies of opioid consumption is a useful addition to defined daily doses. Palliat Med 2011;25:725-32.
20 WHO Collaborating Centre for Drug Statistics Methodology 19th WHO Model List of Essential Medicines. http://www.who.int/medicines/publications/essentialmedicines/ EML2015_8-May-15.pdf?ua=1/ (accessed 8 Jul 2016).

21 Pan HH, Ho ST, Lu CC, et al. Trends in the consumption of opioid analgesics in Taiwan from 2002 to 2007: a population-based study. J Pain Symptom Manage 2013; $45: 272-8$

22 De Lima L, Pastrana T, Radbruch L, et al. Cross-sectional pilot study to monitor the availability, dispensed prices, and affordability of opioids around the globe. J Pain Symptom Manage 2014;48:649-59.

23 Ministry of human resources and social security. Notice concerning the catalogue of basic medical insurance, injury insurance and maternity insurance for the issuing country. http://www.mohrss.gov.cn/yiliaobxs/YILIAOBXSzhengcewenjian/200911/ t20091127_83725.html (accessed 8 Jul 2016).

24 Yang YP, Ma YX, Huang Y, et al. The good pain management (GPM) ward program in China and its impact on Chinese cancer patients: the SYSUCC experience. Chin J Cancer 2014;33:323-9.

25 Maltoni M, Scarpi E, Modonesi C, et al. A validation study of the WHO analgesic ladder: a two-step vs three-step strategy. Support Care Cancer 2005;13:888-94.

26 Bandieri E, Romero M, Ripamonti Cl, et al. Randomized trial of low-dose morphine versus weak opioids in moderate cancer pain. J Clin Oncol 2016;34:436-42.

27 NCCN. The NCCN Adult cancer pain guideline (Version 1.2015) [EB/OL. Fort Washington: NCCN, 2015. (accessed 29 Jul 2016).

28 Caraceni A, Hanks G, Kaasa S, et al. Use of opioid analgesics in the treatment of cancer pain: evidence-based recommendations from the EAPC. Lancet Oncol 2012;13:e58-e68.

29 Hamunen K, Paakkari P, Kalso E. Trends in opioid consumption in the Nordic countries 2002-2006. Eur J Pain 2009;13:954-62.

30 Tassinari D, Sartori S, Tamburini E, et al. Adverse effects of transdermal opiates treating moderate-severe cancer pain in comparison to long-acting morphine: a meta-analysis and systematic review of the literature. J Palliat Med 2008;11:492-501.

31 Riley J, Branford R, Droney J, et al. Morphine or oxycodone for cancer-related pain? A randomized, open-label, controlled trial. J Pain Symptom Manage 2015;49:161-72. 